\title{
Abrupt deterioration and pulmonary embolism in COVID-19: a case report
}

\author{
Author: Manish Motwani ${ }^{\mathrm{A}}$
}

\begin{abstract}
A case is presented highlighting pulmonary embolism as an important complication of COVID-19 and the abruptness with which deterioration and widespread pulmonary infiltrates can develop even after relatively normal initial investigations, illustrating the importance of follow-up. The role of computed tomography in COVID-19 is also discussed.
\end{abstract}

KEYWORDS: COVID-19, pulmonary embolism, pneumonia, deterioration

DOI: $10.7861 /$ clinmed.2020-0307

\section{Case report}

A 52-year-old man attended the medical assessment unit with a 5-day history of dry cough, fever, and dyspnea. He had no preexisting medical conditions. Investigations showed a normal full blood count, normal renal and liver biochemistry, normal troponin, normal clotting profile, and only mildly raised C-reactive protein (CRP). Arterial blood gas sampling including oxygenation was within normal range. A CXR and electrocardiograph were also normal. Same-day PCR nasopharyngeal swab detected severe acute respiratory syndrome coronavirus-2 (SARS-CoV-2) RNA, confirming coronavirus disease-19 (COVID-19). As his symptoms were relatively mild and there were no high-risk comorbidities, he was discharged home with virtual clinic follow-up (daily telephone assessments).

Three days later he was significantly more breathless and was advised to re-attend - his oxygen saturation was now $83 \%$ on air. Computed tomography (CT) pulmonary angiography showed widespread bilateral ground-glass opacities consistent with extensive COVID-19 pneumonitis and substantial bilateral subsegmental pulmonary emboli (Fig 1; see also video at https:// youtu.be/frh28BOkhtg). Blood tests now showed the typical COVID-19 profile with mild lymphopenia, mildly raised alanine aminotransferase and lactate dehydrogenase, modestly raised CRP, normal procalcitonin, raised ferritin and substantially raised

Author: ${ }^{A}$ consultant cardiologist, Manchester Heart Centre, Manchester, UK and University of Manchester, Manchester, UK
D-dimer. He was treated with supportive care, high-flow oxygen, and direct oral anticoagulant; fortunately, he did not require mechanical ventilation or intensive care unit management. After 10 days he had recovered sufficiently to be discharged home.

\section{Discussion}

This case highlights the abruptness with which deterioration and widespread pulmonary infiltrates can develop in COVID-19 even after relatively normal initial investigations. It also highlights pulmonary embolic disease as an important complication, reported in up to $33 \%$ of cases..$^{1,2}$ it is uncertain if the etiology is pulmonary thrombosis, thrombo-embolism or part of cytokinestorm-driven disseminated intravascular coagulation. ${ }^{2}$ Further studies are required to understand the pathophysiology behind this complication of COVID-19 and guide universally agreed anticoagulation policies.

Regarding the role of CT in COVID-19, professional societies have cautioned against its routine use as a first-line diagnostic test - because although highly sensitive, the CT features of COVID-19 pneumonia are not specific and also tend to peak later (day 6-11). ${ }^{3}$ Importantly, indiscriminate use of CT also places healthcare workers (eg technologists) at risk of exposure. Accordingly, most suspected COVID-19 patients are diagnosed on the basis of clinical assessment, CXR and blood tests while awaiting PCR results.

Nonetheless, with recognition of poor PCR sensitivity, many centres adopt the pragmatic approach of using $C T$ in cases with the combination of high clinical suspicion, normal or indeterminate CXR, and negative PCR result, and in patients with marked deterioration in order to exclude occult pulmonary embolism. ${ }^{3}$ However, as decontamination and patient segregation protocols become more embedded in hospitals, the risk-benefit ratio may ultimately support wider diagnostic use. New CT technology and advances in post-processing may also support further application. For example, we highlight how simple 3D-volume rendering and semi-automated segmentation can provide a rapid visual map of inflammatory burden and the extent of disease, but this can be taken further: artificial intelligence methods have already been utilised to fully quantify opacity burden (facilitating response to treatment assessments) and distinguish findings in COVID-19 from community-acquired pneumonia or other lung disease. Such tools may prove indispensable in a prolonged pandemic or during a second wave. ${ }^{4}$ 

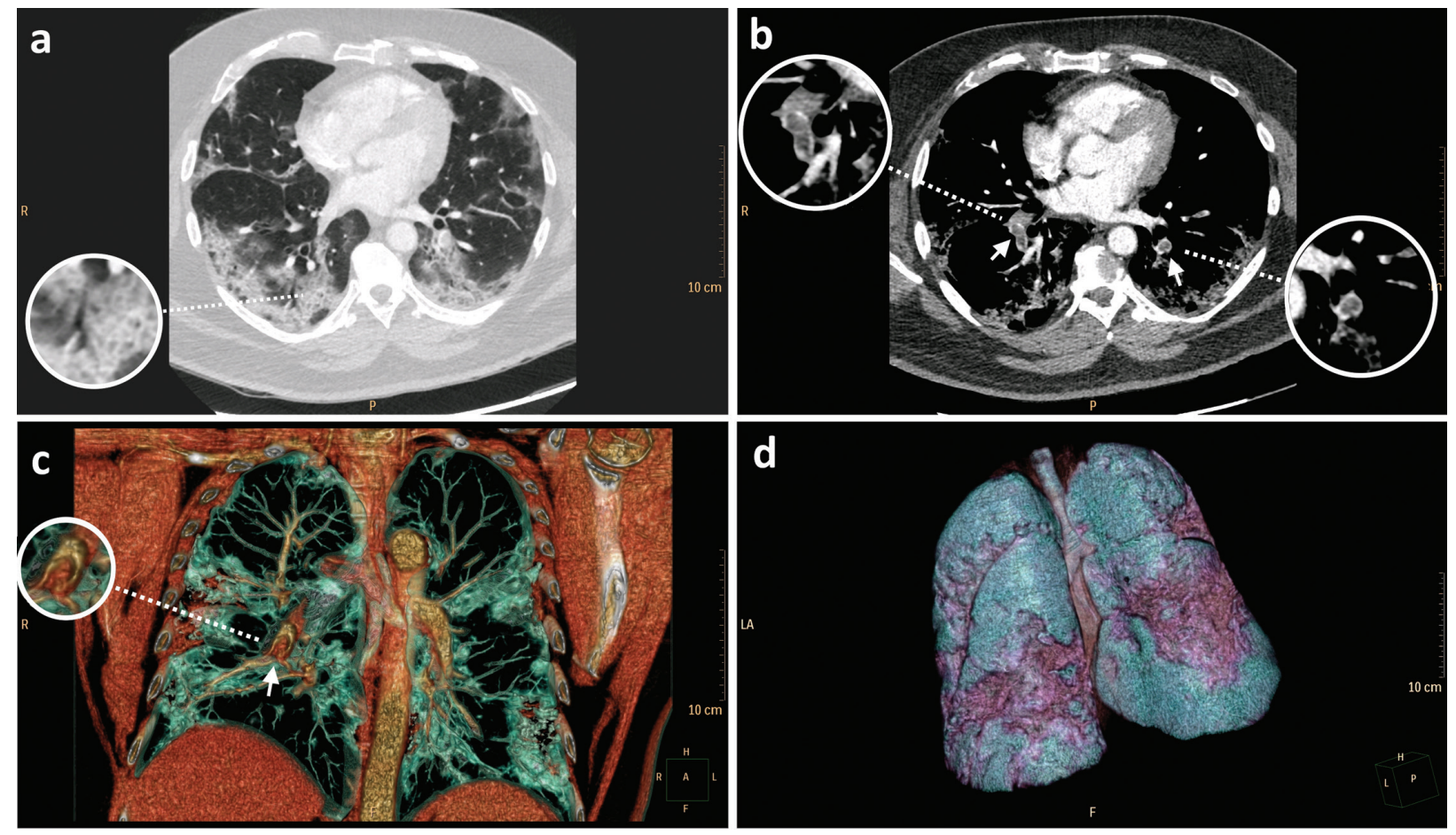

Fig 1. Chest computed tomography pulmonary angiography and three-dimensional visualisation. a) Lung windowing shows bilateral peripheral ground-glass opacities consistent with COVID-19. b) Angiographic windowing shows bilateral subsegmental pulmonary emboli (arrows). Three-dimensional volume-rendering of thickened coronal slab (c; abnormal lung is green; pulmonary embolus also visualised, arrow) and of whole-data segmented lung parenchyma (d; abnormal lung is purple) helps visually demonstrate the widespread inflammatory burden.

\section{References}

1 Grillet F, Behr J, Calame P et al. Acute pulmonary embolism associated with COVID-19 pneumonia detected by pulmonary CT angiography. Radiology 2020, in press (doi: 10.1148/ radiol.2020201544).

2 Poissy J, Goutay ], Caplan M et al. Pulmonary embolism in COVID-19 patients: awareness of an increased prevalence. Circulation 2020, in press (doi: 10.1161/ CIRCULATIONAHA.120.047430).

3 Tavare AN, Braddy A, Brill S et al. Managing high clinical suspicion COVID-19 in patients with negative RT-PCR: a pragmatic and limited role for thoracic CT. Thorax 2020, in press (doi: 10.1136/ thoraxjnl-2020-214916).

4 Kundu S, Elhalawani H, Gichoya JW, Kahn Jr CE. How might $\mathrm{AI}$ and chest imaging help unravel COVID-19's mysteries? Radiology Artificial Intelligence 2020, in press (doi: 10.1148/ ryai.2020200053)

Address for correspondence: Dr Manish Motwani, Manchester Heart Centre, Manchester University NHS Foundation Trust, Oxford Road, Manchester M13 9WL, UK.

Email: mani.motwani@mft.nhs.uk 